\title{
Control of Internal Induction of Galactose Pathway Enzymes in an Escherichia coli Mutant
}

\author{
E. JORDAN* AND M. B. YARMOLINSKY \\ McCollum-Pratt Institute and Department of Biology, \\ The Johns Hopkins University, Baltimore, Maryland, U.S.A.
}

(Received 7 June 1962)

\begin{abstract}
SUMMARY
Galactokinaseless mutants of Escherichia coli are, in general, phenotypically constitutive for the remaining enzymes of the galactose pathway, a phenomenon which has been attributed to internal induction (Jordan, Yarmolinsky \& Kalckar, 1962). One exceptional, kinaseless, mutant, though constitutive during stationary phase, is inducible during logarithmic growth. This mutant, w3092A, also produces a constitutive concentration of enzyme after confluent growth on solid medium and continues to do so even after 3-5 generations of subsequent logarithmic growth in liquid medium. This effect of growth on solid medium is peculiar to w3092A. It does not occur in other inducible strains, including galactosefermenting revertants of w3092A, nor in w3092A itself grown as isolated colonies. Even in confluently grown organisms it is lost after storage at $4^{\circ}$ for several days. In each case high degrees of enzyme synthesis can be prevented by an inhibitor of induction of the galactose enzymes, methyl$\beta$-D-thiogalactoside, and are therefore attributed to internal induction. Though the enzyme concentrations decrease rapidly when the cultures resume logarithmic growth after stationary phase, high concentrations may persist up to the fifth generation after confluent growth on solid medium and then decline to reach the basal level only after the 12th generation. The inducibility of w3092A (A character) was shown to be independent of the mutation to kinaseless, since other kinaseless mutants derived from a galactose-fermenting revertant of w3092A still carried the A character.
\end{abstract}

\section{INTRODUCTION}

Galactose is converted to glucose in Escherichia coli by a series of inducible enzymes determined by genes located in the 'gal region' of the chromosome. Three enzymes and their corresponding cistrons have been identified (Morse, Lederberg \& Lederberg, 1956a, b; Kurahashi, 1957; Kalckar, Kurahashi \& Jordan, 1959; Lederberg, 1960; Soffer, 1961). These catalyse the reactions described by the equations given below:

$$
\begin{aligned}
& \text { Gal + ATP } \underset{\text { cistron A }}{\stackrel{\text { galactokinase }}{\longrightarrow} \text { Gal-1-P + ADP }} \\
& \text { Gal-1-P + UDPG } \underset{\text { cistron B }}{\stackrel{\text { transferase }}{\rightleftarrows}} \text { UDPGal + G-1-P } \\
& \text { UDPGal } \underset{\text { cistron D }}{\stackrel{\text { epimerase }}{\rightleftharpoons}} \text { UDPG }
\end{aligned}
$$

* Present address : Biological Laboratories, Harvard University, Cambridge, Massachusetts, U.S.A. 
The following abbreviations are used: gal, D-galactose; gal-1-P, D-galactose-1phosphate; G-1-P, $\alpha$-D-glucose-1-phosphate; UDPG, uridine diphosphoglucose; UDPGal, uridine diphosphogalactose; NAD, nicotinamide-adenine dinucleotide; NADH, reduced NAD; ADP, adenosine diphosphate; ATP, adenosine triphosphate; galactokinase or kinase, ATP:D-galactose-1-phosphotransferase (E.C. 2.7.1.6); transferase, UDPglucose: $\alpha$-D-galactose-1-phosphate uridylyltransferase (E.C. 2.7.7 .12); epimerase, UDPglucose 4-epimerase (E.C. 5.1.3.2); UDPG pyrophosphorylase, UTP: $\alpha$-D-glucose-1-phosphate uridylyltransferase (E.C. 2.7.7.9).

It was recently shown also that lack of the enzyme UDPG pyrophosphorylase results in an inability to metabolize galactose (Fukasawa, Jokura \& Kurahashi, 1962). The gene controlling this enzyme is not located in the 'gal region'-nor is the enzyme inducible by galactose. An operator locus within the 'gal region' (Kalckar et al. 1959; Lederberg, 1960; Buttin, 1961) and a repressor locus outside this region responsible for the control of the galactose enzymes (Buttin, 1961) have also been identified.

It has been shown that the majority of kinaseless mutants are constitutive for the remaining enzymes of the galactose sequence and that revertants to galactose fermentation are again inducible. In this case constitutive synthesis can be inhibited by methyl- $\beta$-D-thiogalactoside (MTG), an inhibitor of induction for the galactose enzymes (Buttin, 1961). Constitutivity associated with lack of kinase was therefore attributed to internal induction by a substance which in the wild type is inactivated by a functional kinase (Jordan et al. 1962). The induction achieved by the addition of $\mathbf{D}$-galactose or $\mathbf{D}$-fucose corresponds to an increase in enzyme concentration of only a few fold; internal induction may be responsible for the high basal enzyme concentration even in the wild type.

One exceptional, inducible, kinaseless strain was found in our laboratory (Yarmolinsky \& Wiesmeyer, 1959). This strain, w3092A, though inducible during logarithmic growth, is phenotypically constitutive in stationary phase. We previously reported (Yarmolinsky, Jordan \& Wiesmeyer, 1961) a phage-produced alteration of enzyme inducibility in this strain. It was found that treatment of w3092A with phage, to effect prophage substitution, resulted in a change to constitutive enzyme production in logarithmic growth phase. Subsequent work has shown, however, that our original interpretation was erroneous. In these experiments one uncontrolled variable was systematically introduced: the type of colonial growth from which inocula were taken for the growth of assay cultures. It appears that a physiological modification, which may last for many generations, takes place in w3092A during confluent growth on solid medium. The nature of this modification is the subject of this paper.

\section{METHODS}

Materials and methods, including strains of Escherichia coli $\mathbf{k}-12$, were essentially as described in Jordan et al. (1962).

Growth media. Tryptone broth: $1 \%$ Difco Bacto-Tryptone with $0.5 \% \mathrm{NaCl}$. $1.5 \%$ agar was added for tryptone agar.

Minimal agar: medium A (Hartman, 1956). A salts medium with $\mathrm{NH}_{4}{ }^{+}$as nitrogen source and $1 \%$ glucose as carbon source-with $1.5 \%$ agar. 
EMB agar: eosin-methylene blue agar (Campbell, 1957). $1 \%$ galactose was added to make EMB gal agar.

Enzyme assay. The assay for epimerase, a modification of the method of Kalckar et al. (1959) was performed on lysozyme-disrupted organisms as follows. About $10^{7}$ organisms were mixed in the cuvette with an equal volume of a lysis mixture (K. Paigen, personal communication) $0.3 \mathrm{mg}$ lysozyme $/ \mathrm{ml}$. in $0 \cdot 1 \mathrm{M}$-tris(hydroxymethyl)aminomethane-acetate ( $\mathrm{pH} \mathrm{8.0)}$, containing $7.5 \times 10^{-3} \mathrm{M}$-ethylenediaminetetraacetate. The reaction mixture, consisting of $100 \mu$ moles glycine (pH 8.7) 0.05 $\mu$ mole UDPGal (Wiesmeyer \& Jordan, 1961), 2.5 $\mu$ mole NAD and 100 units (Strominger, Maxell, Axelrod \& Kalckar, 1957) of UDPG dehydrogenase, was added to the lysed organisms immediately. It was found that cells prepared in this way had negligible NADH oxidase activity.

Most of the assays reported in this paper were performed by the above method. In these cases, specific activity was calculated using optical density of the suspension of organisms at $650 \mathrm{~m} \mu$ as a measure of protein. To obtain units comparable to those previously published, it was estimated that an optical density at $650 \mathrm{~m} \mu$ of $5 \cdot 0$ corresponded to $1.0 \mathrm{mg}$. protein $/ \mathrm{ml}$. Thus the unit used here, $\mu$ mole UDPG/hr/ o.D.650 = 5, is roughly equivalent to $\mu$ mole UDPG $/ \mathrm{hr} . / \mathrm{mg}$. protein, the unit used previously.

\section{RESULTS AND DISCUSSION}

For some time we were puzzled that the conversion of Escherichia coli, w3092A to the constitutive state occurred only during prophage substitution experiments. A close scrutiny of the protocols for such experiments finally revealed only one variable which correlated with conversion to constitutivity-the type of growth from which the inoculum for the assay cultures was taken. It seemed highly unlikely that differences in the origin of the inoculum could account for our findings, but a compilation of all relevant data seemed to bear out the hypothesis. This is presented in Table 1. It is seen that, under the conditions described, in about four

Table 1. Effect of origin of inoculum on epimerase inducibility in strain w3092A

\begin{tabular}{|c|c|c|c|c|}
\hline \multirow[b]{2}{*}{ Solid medium } & \multicolumn{2}{|c|}{ Confluent colonies } & \multicolumn{2}{|c|}{ Isolated colonies } \\
\hline & $\begin{array}{c}\text { No. } \\
\text { constitutive }\end{array}$ & $\begin{array}{c}\text { No. } \\
\text { inducible }\end{array}$ & $\begin{array}{c}\text { No. } \\
\text { constitutive }\end{array}$ & $\begin{array}{c}\text { No. } \\
\text { inducible }\end{array}$ \\
\hline EMB & 48 & 12 & $\mathbf{0}$ & 28 \\
\hline Tryptone & 16 & $\mathbf{5}$ & $\mathbf{0}$ & 5 \\
\hline Minimal glucose & 2 & $\mathbf{0}$ & $\mathbf{0}$ & 6 \\
\hline
\end{tabular}

Epimerase assays were performed on liquid cultures after about three generations of growth in the presence or absence of $10^{-2} \mathrm{M}$-galactose as inducer. The optical density at $650 \mathrm{~m} \mu$ of the freshly inoculated cultures was between $0 \cdot 01$ and $0 \cdot 02$. Constitutive and inducible are defined as a specific activity ratio of + gal/ - gal grown cultures of $<2.5$ and $\geqslant 2.5$ respectively.

out of five cases, cultures derived from confluent colonies behave as if they were constitutive, while cultures derived from isolated colonies are inducible. While results for epimerase only are reported here, experiments in which transferase was assayed indicate that this enzyme behaves similarly. Furthermore, the method of cell disruption appears to be immaterial. Initial experiments 'Yarmolinsky et al 
1961) employed sonication and yielded results comparable to those obtained later with lysozyme.

With this encouragement, the experiment shown in Table 2 was performed. A culture of w3092A was streaked on EMB agar to obtain isolated colonies. Some of these (column A) were used directly as inocula for cultures to be assayed, others were first restreaked on EMB plates to obtain confluent growth (column B). Clearly all strains under column $\mathbf{A}$, derived from isolated colonies, are inducible, while all except one under column B, derived from confluent colonies, are constitutive.

Table 2. Effect of origin of inoculum on epimerase levels in strain $\mathrm{w3092 \textrm {A }}$ and its reversibility on solid medium

\begin{tabular}{|c|c|c|c|c|c|}
\hline $\begin{array}{l}\text { Solid medium.... } \\
\text { Type of growth... }\end{array}$ & $\begin{array}{c}\text { A } \\
\text { EMB } \\
\text { Isolated }\end{array}$ & $\begin{array}{c}\text { B } \\
\text { EMB } \\
\text { Confluent }\end{array}$ & $\begin{array}{c}\text { C } \\
\text { EMB } \\
\text { Isolated }\end{array}$ & $\begin{array}{l}\text { D } \\
\text { Min glu } \\
\text { Isolated }\end{array}$ & $\begin{array}{c}\mathrm{E} \\
\text { Min glu* } \\
\text { Confluent }\end{array}$ \\
\hline \multirow{5}{*}{$\begin{array}{l}\text { Specific activity of } \\
\text { epimerase in broth } \\
\text { culture }\end{array}$} & $4 \cdot 2 / 16$ & $16 / 11$ & $\left\{\begin{array}{l}3 \cdot 2 / 14 \\
1 \cdot 7 / 10\end{array}\right.$ & $\begin{array}{l}3 \cdot 4 / 15 \\
2 \cdot 8 / 13\end{array}$ & $11 / 6 \cdot 7$ \\
\hline & $3 \cdot 4 / 15$ & $13 / 14$ & - & $\begin{array}{l}2 \cdot 3 / 11 \\
2 \cdot 8 / 9 \cdot 5\end{array}$ & $\begin{array}{c}6 \cdot 1 / 5 \cdot 7 \\
-\end{array}$ \\
\hline & 一 & $17 / 13$ & 一 & $\begin{array}{l}1 \cdot 3 / 6 \cdot 5 \\
2 \cdot 1 / 8 \cdot 4\end{array}$ & - \\
\hline & $4 \cdot 0 / 17$ & $13 / 19$ & $\left\{\begin{array}{l}4 \cdot 0 / 15 \\
2 \cdot 7 / 15\end{array}\right.$ & - & - \\
\hline & $\begin{array}{l}4 \cdot 0 / 16 \\
3 \cdot 2 / 17\end{array}$ & $\begin{array}{l}17 / 16 \\
4 \cdot 6 / 15\end{array}$ & - & & - gal \\
\hline
\end{tabular}

The numbers are specific activities of epimerase after three generations growth in tryptone broth (expressed as $\mu$ mole UDPG/hr./o.D.650 $=5$ ). The numerator (denominator) of each ratio are the values obtained when $10^{-3} \mathrm{M}$-galactose was absent (present) in the broth culture. The source of the inoculum for each broth culture is indicated by the column headings. The cultures described in column B were derived from those described in column A by picking from the same plate and streaking out on EMB agar to obtain isolated colonies. Similarly, the cultures of columns C, D, E were derived by picking from the confluently grown column $B$ cultures on to the indicated media.

* It appears from the data in this column that there is no inhibition of enzyme synthesis by glucose in this strain, W3092A. A glucose effect is observed in liquid cultures (Jordan et al. 1962). Its absence here may be due to depletion of glucose in the medium.

It was of interest to find out whether the phenomenon was reversible and whether it was medium-dependent. Conceivably the medium could contain a repressor of enzyme synthesis to which strain w3092A is sensitive and which is locally depleted by confluently growing cells. Such a repressor might not be present in minimal salts medium. Loopfuls were therefore picked from the streaks of column $\mathbf{B}$ and restreaked to obtain isolated or confluent colonies on EMB and minimal glucose agars. Column $\mathrm{C}$ indicates that the phenomenon is fully reversed by growth as isolated colonies. Columns D and E show that minimal solid medium gives essentially the same results as enriched medium. It is therefore unlikely that a repressor from the medium is involved.

Galactose might be formed endogenously in strain w3092A from UDPG by reversal of the reactions catalysed by epimerase and transferase and cleavage of the gal-1-P. Under conditions of confluent growth it might accumulate in this way, causing induction, while in the case of isolated colonies it could diffuse into the medium. As pointed out above, w3092A increases its level of epimerase when grown 
into stationary phase in liquid medium as it does in confluent growth on a solid medium. This may also represent internal induction operating in w3092A during post-logarithmic growth.

Internal induction has been shown to occur in other kinaseless mutants previously studied (Jordan et al. 1962), in both post-logarithmic and logarithmic growth phases by demonstrating that MTG could prevent constitutive enzyme synthesis. The results of a comparable experiment with strain w3092A are shown in Table 3. Suspensions of organisms from confluent growths on EMB or tryptone agar have high concentrations of enzyme even though no galactose is added. However, when MTG is present in the agar, or in liquid medium during subsequent growth of the culture, the enzyme level becomes low. Similarly, the increase in enzyme concentration on going from logarithmic to stationary phase is prevented by MTG. The results therefore bear out the hypothesis that constitutivity in $\mathrm{w}^{3092 \mathrm{~A}}$ is the result of internal induction in each case.

Table 3. Reversal of constitutivity in strain $\mathrm{w3092 \textrm {A }}$ by the inhibitor of induction, methyl- $\beta$-D-thiogalactoside (MTG)

\begin{tabular}{|c|c|}
\hline $\begin{array}{l}\text { Specific activity of } \\
(\mu \text { mole UDPG/hr. } / \mathrm{o} .\end{array}$ & $\begin{array}{l}\text { merase } \\
350=5 \text { ) }\end{array}$ \\
\hline $\begin{array}{c}+10^{-2} \mathbf{M -}^{-} \\
\text {gal* }^{*}\end{array}$ & $\begin{array}{l}+10^{-2} \mathrm{M}- \\
\text { MTG* }^{*}\end{array}$ \\
\hline
\end{tabular}

Confluent colonies

Logarithmic cultures derived from confluent colonies

Cultures derived from a slant

\section{On EMB agar}

On Tryptone agar

On Tryptone agar + MTG

On EMB agar

On Tryptone agar

log. phase

post-log phase gal*

14

14

14

13

1.0

$1 \cdot 0$

$8 \cdot 8$

$7 \cdot 4$

$$
\mathbf{9} \cdot \mathbf{1}
$$

$6 \cdot 1$

14

$\mathbf{5} \cdot 7$

1.7

25

$\begin{array}{cc}- & - \\ - & - \\ - & - \\ - & - \\ - & - \\ 7 \cdot 2 & 0 \cdot 8 \\ 9 \cdot 7 & 0 \cdot 4 \\ 8 \cdot 4 & 1 \cdot 0 \\ 9 \cdot 1 & 1 \cdot 0 \\ 19 & 2 \cdot 3 \\ 18 & 1 \cdot 9 \\ 6 \cdot 3 & 1 \cdot 6 \\ 17 & 1 \cdot 7 \\ 17 & 1 \cdot 7\end{array}$

For assay of confluent colonies, cell suspensions were made in tryptone broth. Logarithmic cultures refers to cells grown about three generations in tryptone broth.

* Galactose or MTG were present during growth in broth.

It should be noted that cultures derived from confluent growth on a stock slant are not constitutive. This appears to be related to the age of such slants. It has been found that after several days storage at $4^{\circ}$, confluently growing organisms no longer are constitutive after growth in broth. Previous to experiments involving prophage substitution (Yarmolinsky et al. 1961) the inocula for the growth of w3092A cultures had been taken from slants stored several days in the cold. Since freshly made solid cultures of w3092A were first used routinely in the course of the prophage substitution experiments, we observed the dependence of enzyme concentration on the growth-history of the inoculum at this time, and mistakenly attributed the alteration in enzyme concentration to the treatment with prophage. 
The high enzyme concentrations observed after internal induction on solid medium may be retained through many generations of logarithmic growth, as illustrated in Fig. 1. A suspension of confluently grown organisms from EMB agar was inoculated into tryptone broth with or without MTG and maintained in logarithmic growth for 15 generations by successive dilution every third generation. It is seen that in the absence of MTG the specific activity of epimerase remained high for about 5 generations and then decreased slowly to reach a stable, low level after 12 generations. In the presence of MTG the enzyme activity decreased more rapidly reaching after only three generations a level equal to the lowest level attained in the

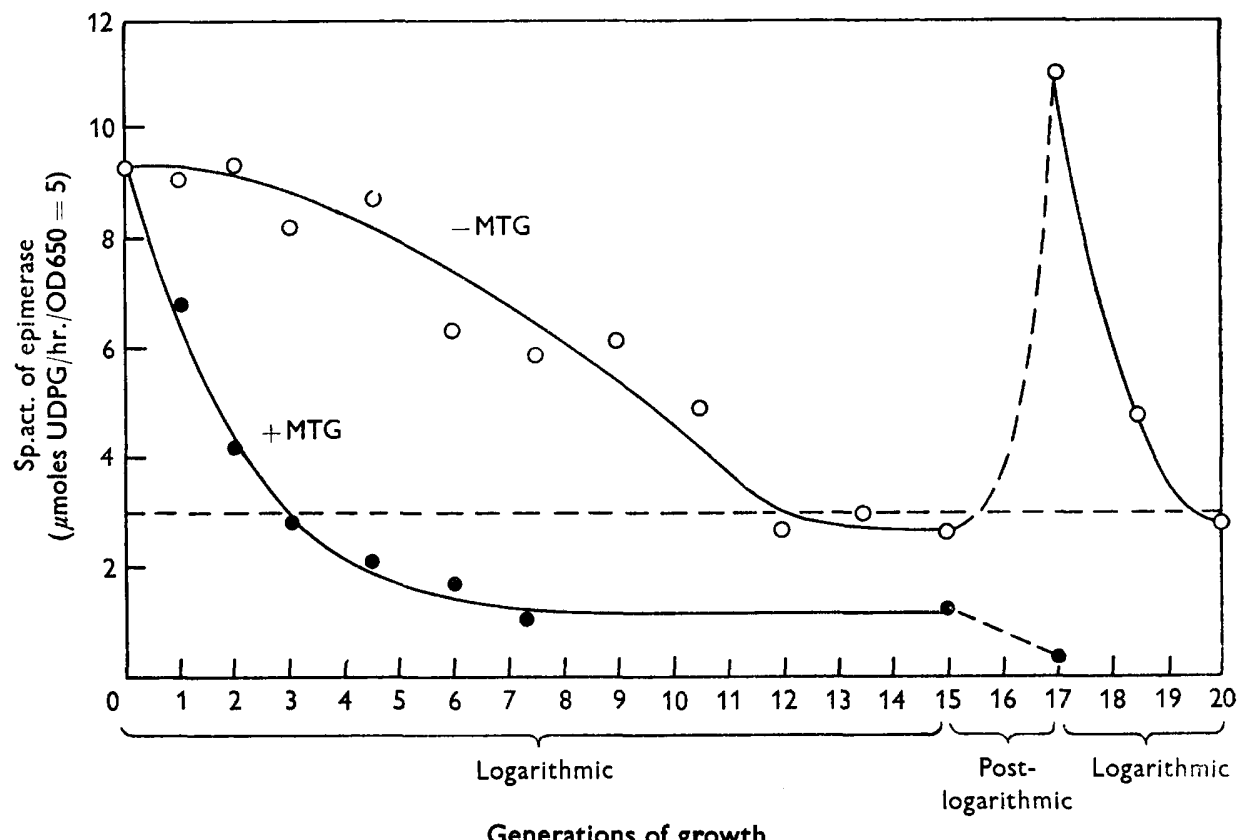

Fig. 1. Kinetics of decrease of epimerase activity after internal induction. A suspension of cells was made from confluent colonies on EMB agar and inoculated into broth $\pm 10^{-2}$ M-MTG. At the end of the $3 \mathrm{rd}, 6 \mathrm{th}, 9$ th and 12th generations the cultures were diluted tenfold to maintain them in logarithmic growth. After the 15th generation the cultures were allowed to go into post-logarithmic phase (until an $0 . \mathrm{D.650}$ of 1.0 was attained) and then diluted to resume logarithmic growth. ...., dashed horizontal line represents the average basal enzyme level in this strain during logarithmic growth in tryptone broth.

absence of MTG and finally levelling off at about one-half this value. This indicates that in this particular strain there is a slight degree of internal induction even in logarithmic phase.

After the fifteenth generation the cultures were allowed to overgrow until an optical density at $650 \mathrm{~m} \mu$ of 1.0 was reached. At this point they were again diluted and permitted to resume logarithmic growth. As seen in the figure, in the absence of MTG the enzyme concentration rises sharply during post-logarithmic growth. If the cells are allowed to continue post-logarithmic growth for longer times than in the experiment illustrated in Fig. 1, concentrations of epimerase two- to threefold higher than those attained on solid medium may be obtained. 
Upon suspension of cells in fresh medium after growth into stationary phase, the specific activity of epimerase falls exponentially to the basal level at the rate expected if internal induction ceases immediately upon resumption of logarithmic growth.

Upon suspension of organisms in fresh medium after confluent growth on solid medium, however, the specific activity of epimerase remains high for about five generations and then decreases at a non-exponential rate much slower than that observed after dilution of stationary phase liquid cultures. This result suggests that during confluent growth on solid medium internal inducer accumulates to a concentration requiring several generations of growth to be diluted out below the threshold concentration required for induction.

The observation, that in stationary phase cells epimerase concentrations are initially higher and yet decrease more rapidly upon suspension in fresh medium than in cells grown confluently on solid medium, seems paradoxical. It may be that growth conditions affect the synthetic capacity of the culture or the rate of elimination of the presumed internal inducer.

\section{Table 4. Independence of the A character and lack of galactokinase}

\begin{tabular}{|c|c|c|c|}
\hline \multirow[b]{2}{*}{ Strain } & \multicolumn{3}{|c|}{$\begin{array}{l}\text { Specific activity of epimerase ( } \mu \text { mole } \\
\text { UDPG/hr./o.D.650 = 5) }\end{array}$} \\
\hline & -gal & $+10^{-2} \mathrm{M}-$ gal & + gal/-gal \\
\hline w3092A & 4.2 & 16 & $\mathbf{3 \cdot 8}$ \\
\hline$K^{-}$from $w 3092 A R$ & $2 \cdot 8$ & 10 & $\mathbf{8 \cdot 6}$ \\
\hline - & 4.6 & 15 & $\mathbf{3 . 3}$ \\
\hline Average of other $\mathrm{K}^{-}$strains & - & - & $1 \cdot 0 *$ \\
\hline Average of gal ${ }^{+}$strains & - & - & $7 \cdot 2 *$ \\
\hline
\end{tabular}

Assays were performed on logarithmic phase cultures derived from overnight cultures.

* Calculated from data of Jordan et al. 1962.

If the organisms are grown in broth containing MTG after growth on solid medium, their epimerase level decreases more rapidly than if they are grown in the absence of MTG, but not as rapidly as would be expected if MTG immediately inhibited the induction of epimerase completely. This suggests that MTG acts competitively with the presumed internal inducer and cannot inhibit enzyme synthesis completely until this inducer has been diluted out by growth.

Strain w3092A arose spontaneously in our laboratory from another kinaseless strain, w3092, which appears constitutive in all growth phases. This suggests that expression of inducibility in $\mathrm{w}^{3092 \mathrm{~A}}$ is the result of a mutation other than that leading to loss of kinase activity. One would therefore expect that other kinaseless strains derived from a galactose-fermenting revertant of w3092A should be inducible. This was found to be the case. The revertant w3092AR (described in Jordan et al. 1962) was treated with $0.02 \% 2$-amino purine according to Demerec (1960). Of the galactose-negative mutants obtained, two were kinaseless by the following criteria. They were transducible to galactose fermentation at high frequency by wild-type transducing phage, but only at low frequency by transducing phage from w3092A. The gal + colonies obtained at low frequency with w3092A are presumably the result of crossing over within the cistron. Furthermore, phage lysates from the 
presumptive kinaseless mutants yielded many more gal + colonies on epimeraseless or transferaseless indicator strains than on the kinaseless w3092A. The appearance of some gal + colonies in crosses of the new kinaseless strains with w3092A indicates that neither of the new strains carries a mutation identical with that in w3092A.

Epimerase assays were performed on the new kinaseless strains derived from w3092R. Table 4 shows that epimerase in both of these strains is inducible, indicating that the mutation permitting inducibility to be expressed is a character independent of the mutation to kinaseless.

This work was supported by PHS research grant E-3832 from the National Institute of Allergy and Infectious Diseases, Public Health Service, and grant G-14429 from the National Science Foundation and was submitted by Elke Jordan, predoctoral fellow of the National Institutes of Health, in partial fulfilment of requirements for the Ph.D. degree at the Johns Hopkins University.

This is contribution No. $\mathbf{3 7 5}$ of the McCollum-Pratt Institute.

\section{REFERENCES}

Butrin, G. (1961). Some aspects of regulation in the synthesis of the enzymes governing galactose metabolism. Cold Spr. Harb. Symp. quant. Biol. 26, 213.

Campbell, A. (1957). Transduction and segregation in Escherichia coli $\mathbf{k}-12$. Virology, 4,366 .

Demerec, M. (1960). Frequency of deletions among spontaneous and induced mutations in Salmonella. Proc. nat. Acad. Sci., Wash. 48, 1075.

Fukasawa, T., Jokura, K. \& Kurahashi, K. (1962). A new enzymatic defect of galactose metabolism. Biochem. Biophys. Res. Com. 7, 121.

Hartman, P. E. (1956). In Genetic studies with bacteria. Carneg. Instn Wash. Publ. 612.

Jordan, E., Yarmolinsky, M. B. \& Kalckar, H. M. (1962). Control of inducibility of enzymes of the galactose sequence in Escherichia coli. Proc. nat. Acad. Sci., Wash. 48, 32.

Kalckar, H. M., Kurahashi, K. \& Jordan, E. (1959). Hereditary defects in galactose metabolism in Escherichia coli mutants. I. Determination of enzyme activities. Proc. nat. Acad. Sci., Wash. 45, 1776.

KURAHASHI, K. (1957). Enzyme formation in galactose-negative mutants of Escherichia coli. Science, 125, 114.

LEDERBERG, E. M. (1960). Genetic and functional aspects of galactose metabolism in Escherichia coli $\mathbf{k}-12$. Symp. Soc. gen. Microbiol. 10, 115.

Morse, M. L., Lederberg, E. M. \& Lederberg, J. (1956a). Transduction in Escherichia coli $\mathbf{K}-12$. Genetics, 41, 142.

Morse, M. L., Lederberg, E. M. \& Lederberg, J. (1956b). Transductional heterogenotes in Escherichia coli. Genetics, $41,758$.

SoFFER, R. L. (1961). Enzymatic expression of genetic units of function concerned with galactose metabolism in Escherichia coli. J. Bact. 82, 471.

Strominger, J. L., Maxwell, E. S., Axelrod, J. \& Kalckar, H. M. (1957). Enzymatic formation of uridine diphosphoglucuronic acid. J. biol. Chem. 224, 79 .

WiEsmeyer, H. \& Jordan, E. (1961). A simple procedure for the preparation of uridine diphosphogalactose. Anal. Biochem. 2, 281.

YARMolinsky, M. B., Jordan, E. \& WiEsmeYer, H. (1961). Regulatory mechanisms in the synthesis of enzymes of galactose metabolism. Part I. Coordinate repression and de-repression of the 'galactose sequence'. Cold. Spr. Harb. Symp. quant. Biol. 26, 217.

YARMolinskY, M. B. \& WresmeYer, H. (1959). Regulation by coliphage lambda of the expression of the capacity to synthesize a sequence of host enzymes. Proc. nat. Acad. Sci., Wash. 46, 1626. 